\title{
Análisis de pérdidas en semiconductores de potencia generadas por controladores difusos de velocidad en motores de CD sin escobillas
}

\author{
Manuel García-López ${ }^{1}$, Antonio Rosales ${ }^{2}$, Pedro Ponce-Cruz ${ }^{2}$, Arturo Molina ${ }^{2}$, \\ Jose J. Rodriguez Rivas ${ }^{1}$ \\ ${ }^{1}$ Instituto Politécnico Nacional, Ciudad de México, \\ México \\ ${ }^{2}$ Instituto Tecnológico y de Estudios Superiores de Monterrey, Ciudad de México, \\ México \\ \{magarcial, jjrodriguezr\}@ipn.mx, \{antonio.rosales, pedro.ponce, armolina $\} @$ itesm.mx
}

Resumen. La vida útil de los semiconductores de potencia está en un entorno ocasionado por las pérdidas de potencia por conmutación y conducción debido a la variedad de perfiles de temperatura a los que se ven sometidos los semiconductores durante su operación. Satisfacer el desempeño de la aplicación normalmente depende de la estrategia de control. Sin embargo, cumplir el objetivo por el sistemas de control sin considerar las pérdidas que se presentan en la etapa de potencia afectara la eficiencia de la etapa de potencia y disminuirá el tiempo de vida de los semiconductores. Este trabajo analiza las pérdidas que se generan en los MOSFET's de un puente inversor fuente de tensión trifásico, que alimenta al motor de CD sin escobillas, al controlar su velocidad empleando un controlador difuso. Para observar si hay mejora se compara la respuesta del controlador difuso con la de un controlador PID. La implementación de los dos controladores se lleva acabo con la co-simulación (LabVIEW-Multisim). Los resultados de la co-simulación demuestran que el control difuso, comparado con un PID, presenta un mejor desempeño en términos del índice del error cuadrático en el control de la velocidad, además de presentar menos pérdidas de potencia, lo cual garantiza una extensión del tiempo de vida de los semiconductores de potencia.

Palabras clave: control difuso, motor de cd sin escobillas, co-simulación, pérdidas de potencia.

\section{Loss Analysis in Power Semiconductors Generated by Fuzzy Speed Controller in Brushless DC Motors}

Abstract. Lifetime of power semiconductors is in an environment affected by power losses due to switching and conduction modes, as a result the semiconductor 
undergoes variations of temperature profiles during its operation. Satisfying the performance required by semiconductor's application depends of the control strategy. However, the accomplishment of control goals without consideration of power losses in the power stage, affect the efficiency as well as decrease the lifetime of the semiconductors. In this paper, the losses in the MOSFETs of a three-phase voltage source inverter bridge, feeding a brushless DC motor, is analyzed. This three-phase voltage source inverter bridge is used to apply a control of velocity, based on fuzzy logic, in the brushless DC motor. In order to verify the advantages of the proposed fuzzy controller an improvement, the response of the fuzzy controller is compared with that one of a PID controller. The implementation of the two controllers is performed by mean of LabView-Multisim co-simulation. Co-simulation results show that the fuzzy controller has better performance than the PID controller, considering mean squared error (MSE) of the tracking error. In addition, fuzzy controller presents less power losses guarantying an extension of the lifetime of the semiconductor.

Keyword: fuzzy control, brushless dc motor, co-simulation, losses power.

\section{Introducción}

Alta eficiencia, tiempo de vida largo, ruido bajo y una buena relación torque-velocidad son las bondades que han acelerado el uso de motores CD sin escobillas en la industria automotriz, aeroespacial y de aparatos de uso doméstico [1]. Particularmente, el control de la velocidad del motor es un aspecto importante en la mayoría de aplicaciones [2]. La velocidad puede regularse en lazo abierto o en lazo cerrado siendo el control en lazo cerrado el más eficiente pues considera el monitoreo en línea de la velocidad.

Los controladores en lazo cerrado más implementados son PID (Proportional-IntegralDerivative) [3] y su uso en motores CD sin escobillas no es la excepción. Sin embargo, la robustez de los controladores PID ante perturbaciones e incertidumbre en los parámetros de la planta no es buena comparada con la robustez que ofrecen técnicas avanzadas como control difuso.

Las características principales del control difuso son las siguientes [4]: es la alternativa del control convencional cuanto la planta presenta no-linealidades que son difíciles de modelar matemáticamente; A diferencia de los controles PID tradicionales, que se basan en referencias fijas, el funcionamiento de los controladores difusos se basa en tareas que pueden involucrar más de una variable del sistema; pueden trabajar como controles adaptables, incluso controladores PID son mejorados con controles difusos para formar PID adaptables; y su característica más importante es que son controladores capaces de sintetizar información de operadores y reflejar esa experiencia (humana) en el desempeño del controlador.

Asegurar que el motor va mantener la velocidad en el valor deseado, usando control clásico o avanzado, es lo ideal en un contexto de control automático. Sin embargo, el control 
de velocidad de motores $\mathrm{CD}$ requiere de una etapa de potencia (puente $\mathrm{H}$ ), la cual se compone de semiconductores que necesitan ser conmutados de manera adecuada porque la conmutación a altas frecuencias los daña. Por lo tanto, la estimación y el análisis de pérdidas en los semiconductores es un aspecto importante a considerar en la práctica [5], no solo por el calentamiento del semiconductor sino también por la eficiencia en términos de potencia.

Para hacer un análisis detallado de cada elemento que compone el lazo de control de velocidad de un motor de DC se debe considerar la diferente dinámica de los elementos, por ejemplo, la dinámica de la electrónica de potencia (amplificador) es más rápida comparada con la dinámica del motor o el controlador. Hacer una simulación de este tipo de sistemas puede resultar un reto pues el software podría aproximar muy bien la dinámica rápida pero la lenta no o viceversa, o simplemente el software es el ideal para simular sistemas de control pero no circuitos eléctricos.

Co-simulación se entiende como la interacción o combinación de 2 plataformas de simulación diferentes con el objetivo de formar un solo sistema donde exista intercambio de datos entre ambos simuladores [7]. Llevar a cabo la co-simulación del sistema en estudio es la alternativa para analizar por un lado el controlador y por otro la parte eléctrica (motor con amplificador de potencia). Particularmente la co-simulación entre Labview y Multisim es una opción interesante debido a que: Multisim ofrece un estudio detallado de la parte eléctrica debido a que los modelos con que trabaja se acercan mucha a la realidad y permiten un análisis en línea de las pérdidas de potencia; y Labview es idóneo para el diseño de controladores y el monitoreo de variables.

En este trabajo se analizan las pérdidas por conducción y conmutación que se generan al controlar la velocidad de un motor de CD sin escobillas. Se estudian dos controladores, uno difuso y un controlador PID, y se compara su desempeño con respecto al error de regulación y la cantidad de pérdidas que genera en la etapa de potencia. Los controladores se implementan en un entorno de co-simulación Labview-Multisim, el cual permite la estimación en línea de las pérdidas por conmutación y conducción. El desempeño de los controladores se mide utilizando el error cuadrático integral. Los resultados de cosimulación demuestran que el controlador difuso presenta un mejor desempeño con menores pérdidas, comparado con el controlador PID.

\section{Accionamiento del MCDSE}

El accionamiento del MCDSE (Motor de CD Sin Escobillas) propuesto para simular la estimación de las perdidas por conmutación y conducción de componentes semiconductores es mostrado en la figura 1, este es integrado por una fuente de tensión de corriente directa (V), un inversor fuente de tensión trifásico (MOSFET'S: 1, 2, 3, 4, 5 Y 6), un MCDSE, sensores de efecto Hall (HC) y controlador. Para que el rotor gire; la corriente tiene que pasar por las bobinas de estator generando un campo magnético que es perpendicular a la dirección del campo magnético de los imanes permanentes esto es una condición para 
obtener el par máximo; una vez iniciado el movimiento de giro la posición del rotor varia en ese instante, por lo tanto, en cada momento hay que conocer la posición en que se encuentra el rotor para poder excitar las bobinas que corresponda y conseguir el par en el instante determinado.[8]

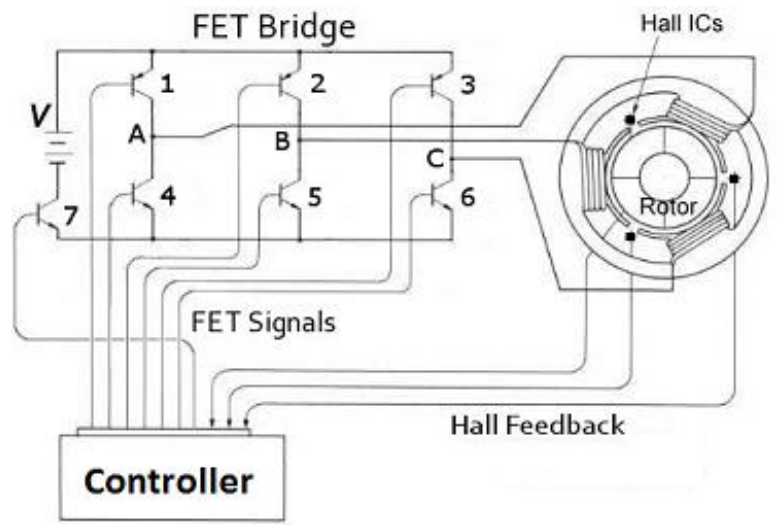

Fig. 1. Esquema general del accionamiento MCDSE.

\subsection{Modelo del MCDSE}

El modelo del MCDSE en espacio de estado es descrito por la ecuación 1.

$$
\dot{\mathrm{x}}=\mathrm{Ax}+\mathrm{Bu},
$$

donde:

$$
A=\left[\begin{array}{ccccc}
-\frac{\mathrm{R}_{\mathrm{s}}}{\mathrm{L}_{1}} & 0 & 0 & -\frac{\lambda_{\mathrm{p}}}{\mathrm{J}} \mathrm{f}_{\mathrm{as}\left(\theta_{\mathrm{r}}\right)} & 0 \\
0 & -\frac{\mathrm{R}_{\mathrm{s}}}{\mathrm{L}_{1}} & 0 & -\frac{\lambda_{\mathrm{p}}}{\mathrm{J}} \mathrm{f}_{\mathrm{bs}\left(\theta_{\mathrm{r}}\right)} & 0 \\
0 & 0 & -\frac{\mathrm{R}_{\mathrm{s}}}{\mathrm{L}_{1}} & -\frac{\lambda_{\mathrm{p}}}{\mathrm{J}} \mathrm{f}_{\mathrm{cs}\left(\theta_{\mathrm{r}}\right)} & 0 \\
\frac{\lambda_{\mathrm{p}}}{\mathrm{J}} \mathrm{f}_{\mathrm{as}\left(\theta_{\mathrm{r}}\right)} & \frac{\lambda_{\mathrm{p}}}{\mathrm{J}} \mathrm{f}_{\mathrm{bs}\left(\theta_{\mathrm{r}}\right)} & \frac{\lambda_{\mathrm{p}}}{\mathrm{J}} \mathrm{f}_{\mathrm{cs}\left(\theta_{\mathrm{r}}\right)} & -\frac{\mathrm{B}}{\mathrm{J}} & 0 \\
0 & 0 & 0 & \frac{\mathrm{P}}{2} & 0
\end{array}\right],
$$




$$
\begin{gathered}
\mathrm{B}=\left[\begin{array}{cccc}
\frac{1}{\mathrm{~L}_{1}} & 0 & 0 & 0 \\
0 & \frac{1}{\mathrm{~L}_{1}} & 0 & 0 \\
0 & 0 & \frac{1}{\mathrm{~L}_{1}} & 0 \\
0 & 0 & 0 & \frac{1}{\mathrm{~J}} \\
0 & 0 & 0 & 0
\end{array}\right] ; \mathrm{L} 1=\mathrm{L}-\mathrm{M}, \\
\mathrm{u}=\left[\begin{array}{llll}
\mathrm{v}_{\mathrm{as}} & \mathrm{v}_{\mathrm{bs}} & \mathrm{v}_{\mathrm{cs}} & \mathrm{T}_{\mathrm{l}}
\end{array}\right]^{\mathrm{T}} .
\end{gathered}
$$

\subsection{Controladores}

Un lazo cerrado se implementa para aumentar la robustez paramétrica a los disturbios. Además, modifican la dinámica de la planta para que se alcancen los criterios de diseño [12] figura 2. Los controladores estudiados son el PID y el difuso

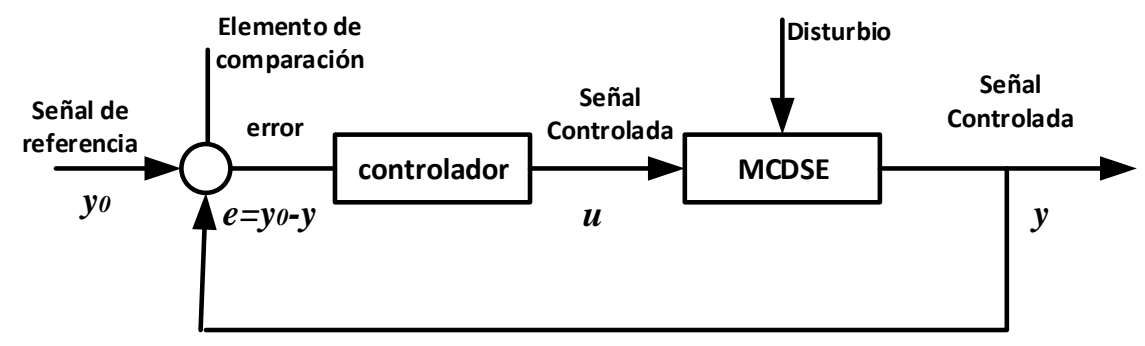

Fig. 2. Esquema del accionamiento del Motor de Corriente Directa Sin Escobillas.

La ecuación (6), es una operación lineal realizada por un Controlador PID convencional; la cual consiste en aplicar propiamente la suma de tres acciones de control: una acción proporcional $\left(\mathbf{e}_{\mathbf{p}}\right)$, una acción integral $\left(\mathbf{e}_{\mathbf{I}}\right)$ y una acción derivativa $\left(\mathbf{e}_{\mathbf{D}}\right)$ [9]:

$$
\mathbf{u}=\mathbf{K}_{\mathbf{P}} \mathbf{e}_{\mathbf{p}}+\mathbf{K}_{\mathrm{I}} \mathbf{e}_{\mathbf{I}}+\mathbf{K}_{\mathrm{D}} \mathbf{e}_{\mathbf{D}}
$$

donde: $\mathbf{K}_{\mathbf{p}}$ - ganancia proporcional, $\mathbf{K}_{\mathbf{I}^{-}}$ganancia integral, $\mathbf{K}_{\mathbf{D}}-$ ganancia derivativa, $\mathbf{e}_{\mathbf{p}}=$ $\mathbf{e}(\mathbf{t}), \mathbf{e}_{\mathrm{I}}=\int_{0}^{t} \mathbf{e}(\tau) \mathbf{y} \mathbf{e}_{\mathrm{D}}=\frac{\mathrm{de}(\mathrm{t})}{\mathrm{dt}}$. 
Un Controlador Difuso consiste en la realización de la estrategia de control en forma cualitativa utilizando lógica difusa para crear un controlador difuso que emula una estrategia heurística de reglas basadas en experiencia o prácticas. La operación no-lineal realizada por un controlador difuso (en donde la fusificación es basada en conjuntos difusos, la operación lógica AND es realizada con el operador Min, el método del centro de gravedad es aplicado para la defusificación) puede ser expresada por la ecuación (7) [9]:

$$
\begin{array}{r}
u=\sum_{i=1}^{4} \sum_{j=1}^{4} \sum_{k=1}^{4} v_{i} w_{j} q_{k}\left(a_{0 i j k}+a_{2 i j k} e_{I}+a_{3 i j k} e_{D}+a_{4 i j k} e_{P} e_{I}\right. \\
\left.+\quad a_{1 i j k} e_{P}+a_{5 i j k} e_{P} e_{D}+a_{6 i j k} e_{I} e_{D}+a_{7 i j k} e_{P} e_{I} e_{D}\right) .
\end{array}
$$

Las variables lógicas $\mathrm{v}_{\mathrm{i}}, \mathrm{w}_{\mathrm{j}}, \mathrm{q}_{\mathrm{k}}$ llevan la información de la señal actual de las membresías a un sector del espacio de entrada, por ejemplo, $v_{i}$ es dada por las relaciones de las ecuaciones (8), (9), (10) y (11)

$$
\begin{aligned}
& \mathrm{v}_{1}=\left\{\begin{array}{lr}
1 & \text { para } \mathrm{e}_{\mathrm{P}} \leq \mathrm{e}_{\mathrm{P} 1}, \\
0 & \text { otro, }
\end{array}\right. \\
& \mathrm{v}_{2}=\left\{\begin{array}{lr}
1 & \text { para } \mathrm{e}_{\mathrm{PI}}<\mathrm{e}_{\mathrm{P}} \leq \mathrm{e}_{\mathrm{P} 2}, \\
0 & \text { otro, }
\end{array}\right. \\
& \mathrm{v}_{3}=\left\{\begin{array}{lr}
1 & \text { para } \mathrm{e}_{\mathrm{P} 2}<\mathrm{e}_{\mathrm{P}} \leq \mathrm{e}_{\mathrm{P} 3} \\
0 & \text { otro }
\end{array}\right. \\
& \mathrm{v}_{4}= \begin{cases}1 & \text { para } \mathrm{e}_{\mathrm{P} 3} \leq \mathrm{e}_{\mathrm{P}}, \\
0 & \text { otro }\end{cases}
\end{aligned}
$$

Las variables $\mathrm{w}_{\mathrm{j}}, \mathrm{q}_{\mathrm{k}}$ pueden ser realizadas de forma similar.

\subsection{Co-simulación}

Los programa de simulación de NI Multisim, desarrollado para modelar y analizar circuitos eléctrico, y LabVIEW, desarrollado para simular y diseñar sistemas de control, son utilizados para realizar la simulación (co-simular) entre el sistema de control y los circuitos electrónicos de potencia. La figura 3, muestra un esquema en detalle de la cosimulación de todo el sistema de prueba. El bloque "Brushless DC Motor VSI Thermal" es un programa en Multisim 2014.0.1 en donde se simulan el inversor fuente de tensión, motor de CD sin escobillas y los sensores de efecto HALL (embebidos en el bloque del motor de CD). El bloque "Brushless DC Motor VSI Thermal" está en un entorno "vi" (virtual instrument) de LabVIEW, en el cual se simulan los controles de velocidad PID y Difuso (a partir del error en el caso del PID y error y cambio del error en el caso del Difuso), así, como la generación de los pulsos de disparo que controlan las compuertas de los MOSFET del puente inversor fuente de tensión [10]. 


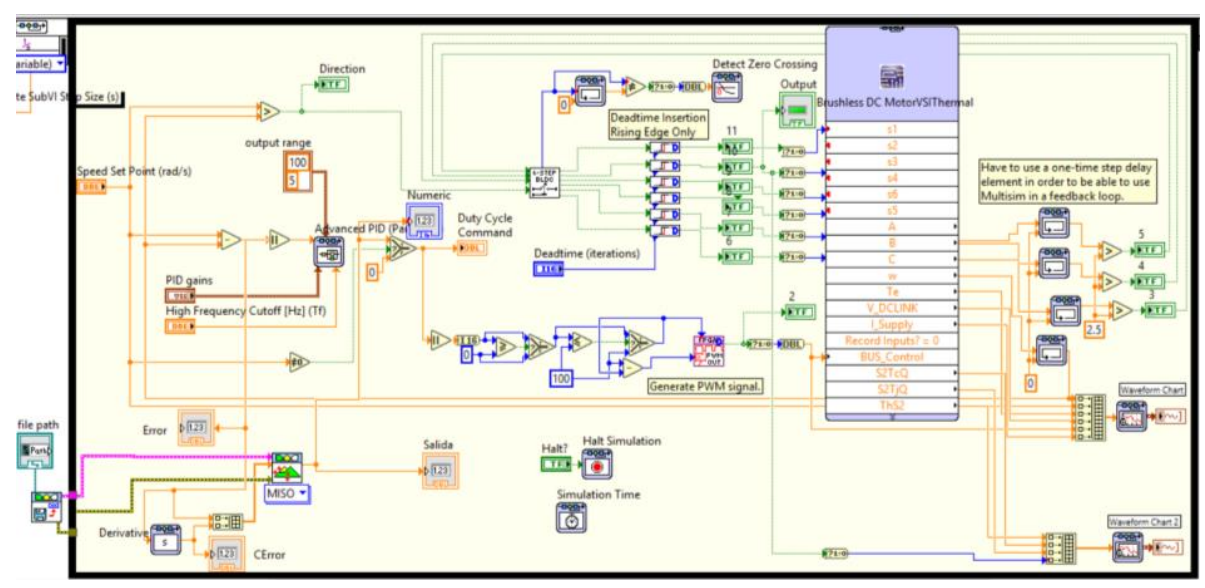

Fig. 3. Detalle del programa de co-simulación entre LabVIEW y MULTISIM [10].

\section{Procedimiento de la implementación}

El propósito de este trabajo es investigar el impacto de un algoritmo de control difuso y compararlo con un algoritmo PID en términos de las perdidas por conducción y conmutación en los MOSFET's de inversor fuente de tensión; por lo que se implementó el esquema general del accionamiento MCDSE de la figura 1. Este esquema se realiza a partir de la co-simulación entre LabVIEW y MULTISIM.

En LabVIEW se implementan los algoritmos del y PID y control difuso. Los parámetros del PID son $\boldsymbol{K}_{\boldsymbol{C}}=500 \operatorname{los} \boldsymbol{K}_{\boldsymbol{I}}, y \boldsymbol{K}_{\boldsymbol{D}}$ son igual 0. Para el control difuso en el proceso de fusificación se tiene las funciones de membresía de la figura 4.
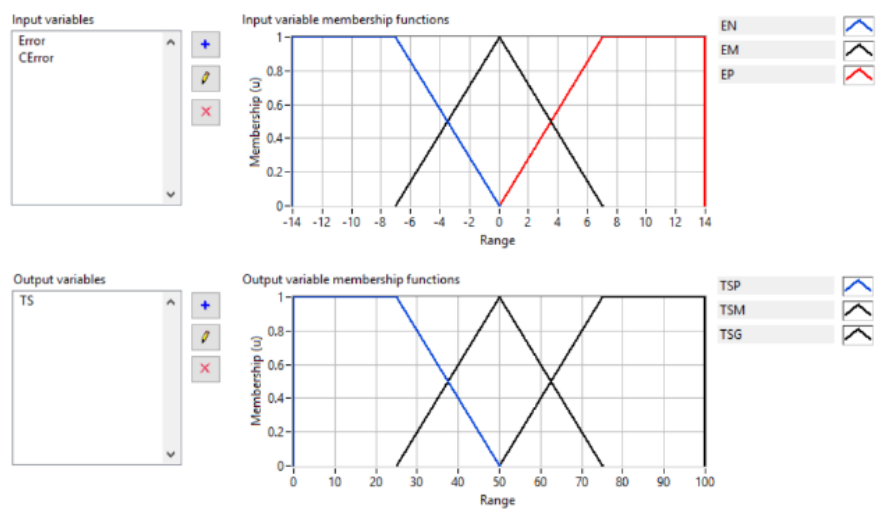

Fig. 4. Realización de la fusificación, funciones de membresía de entrada y salida. 
En la figura 4: Error $=$ VelocidadDeReferencia - VelocidaActual, CError $=$ ErrorAnteror - ErrorActual,$E N=$ ErrorNegativo, $E M=$ ErrorMedio, $E P=$ ErrorPositivo.

Las reglas de inferencia y la estimación de la variable de salida se muestra en la figura 5, por lo que, la salida es obtenida por el método del centro de áreas. En este proceso de defusificación se tienen las funciones de membresía de la figura 4 , en donde: TS = TensionDeSalida, TSP $=$ TensionDeSalidaPequeña, TSM $=$ TensionDeSalidaMedia y TSP $=$ TensionDeSalidaGrande

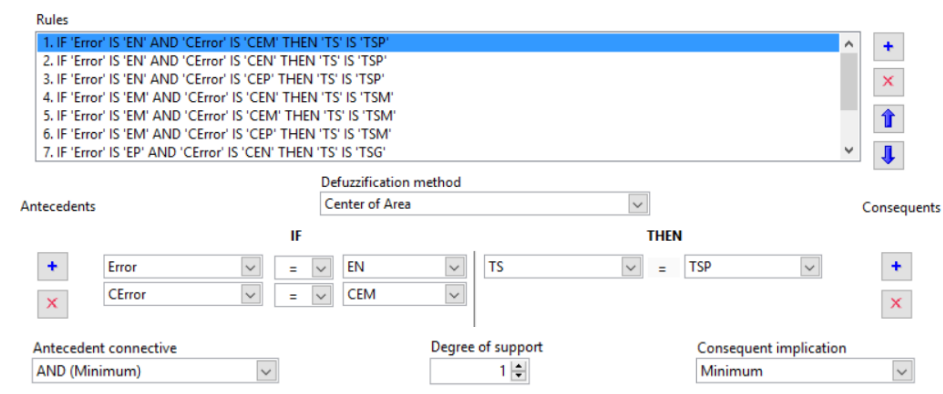

Fig. 5. Realización de la inferencia y defusificación, método por centro de áreas.

Tabla 1. Parámetros del Motor Corriente Directa de Sin Escobillas.

\begin{tabular}{ccc}
\hline & Magnitud & Unidad \\
\hline Inductancia del Estator & 0.15 & $\mathrm{mH}$ \\
Resistencia del Estator & 0.6 & $\Omega$ \\
Constante de velocidad & 0.03 & $\mathrm{Vs} / \mathrm{rad}$ \\
Constante del par & 0.03 & $\mathrm{Nm} / \mathrm{A}$ \\
Numero de par de polos & 1 & \\
\hline
\end{tabular}

En NI Multisim 2014.01se implementan los circuitos electrónicos de potencia: fuente corriente directa, "V", inversos fuente de tensión, "FET bridge" y el motor de CD sin escobillas, MCDSE. La tabla 1, muestra los parámetros del MCDSE; este es adquirido y realizado de acuerdo a los parámetros del componente BRUSHLESS_DC_MACHINE [13]. La tabla 2, muestra los parámetros nominales del MOSFET EPC2015 en estudio [14].

La obtención de las perdidas por conducción y conmutación, del MOSFET EPC2015, es realizada adquiriendo el componente MOSFET_DIODE_THERMA de NI Multisim 2014.0.1; el cual es basado en el modelo de una red electro-térmica la cual es obtenida a partir de la estructura simplificada de los semiconductores MOSFET-Diodo, montados en un casco que a su vez es montado sobre un disipador como lo muestra la figura 6. Las 
potencias por calor en las uniones, que es donde es más grande, son igual al producto $I X V$ en el MOSFET y Diodo, el calor fluye a través de la impedancia térmica de la estructura y se disipa en el entorno ambiente. En el modelo se simplifica el proceso del fuljo del calor en una dimensión, por lo tanto, el recorrido térmico se basa en utilizar agrupaciones de impedancias térmicas en la interface de la superficie entre el casco y el disipador.

La potencia IxV se presenta como una corriente, por lo que se utilizan fuentes de corriente para su representación. La temperatura existente en los puntos físicos de unión de la red térmica, así, como la temperatura ambiente son representada por fuentes de tensión. Las impedancias térmicas son representadas usando elementos R-C, figura 7 [11].

Tabla 2. Valores nominales del MOSFET EPC2015.

\begin{tabular}{|c|c|c|}
\hline & Magnitud & Unidad \\
\hline $\mathbf{V}_{\mathrm{DS}}$ & 40 & V \\
\hline $\mathbf{R}_{\mathrm{DS}(\mathbf{O N})}$ & 4 & $\mathrm{~m} \Omega$ \\
\hline $\mathbf{I}_{\mathbf{D}}$ & 33 & A \\
\hline $\mathbf{T}_{\mathbf{J}}$, temperatura de operación & -40 a 150 & ${ }^{\circ} \mathrm{C}$ \\
\hline $\begin{array}{l}\mathbf{R K}_{\boldsymbol{\theta J C}} \cdot \text { Resistencia térmica. } \\
\text { Unión a casco }\end{array}$ & 2.1 & ${ }^{\circ} \mathrm{C} / \mathrm{W}$ \\
\hline $\begin{array}{l}\mathbf{R K}_{\boldsymbol{\theta} \mathbf{} \mathbf{B}} \text {. Resistencia térmica. } \\
\text { Unión a tarjeta }\end{array}$ & 15 & ${ }^{\circ} \mathrm{C} / \mathrm{W}$ \\
\hline $\begin{array}{l}\mathbf{R K}_{\boldsymbol{\theta J A}} \cdot \text { Resistencia térmica. } \\
\text { Unión al ambiente }\end{array}$ & 54 & ${ }^{\circ} \mathrm{C} / \mathrm{W}$ \\
\hline
\end{tabular}

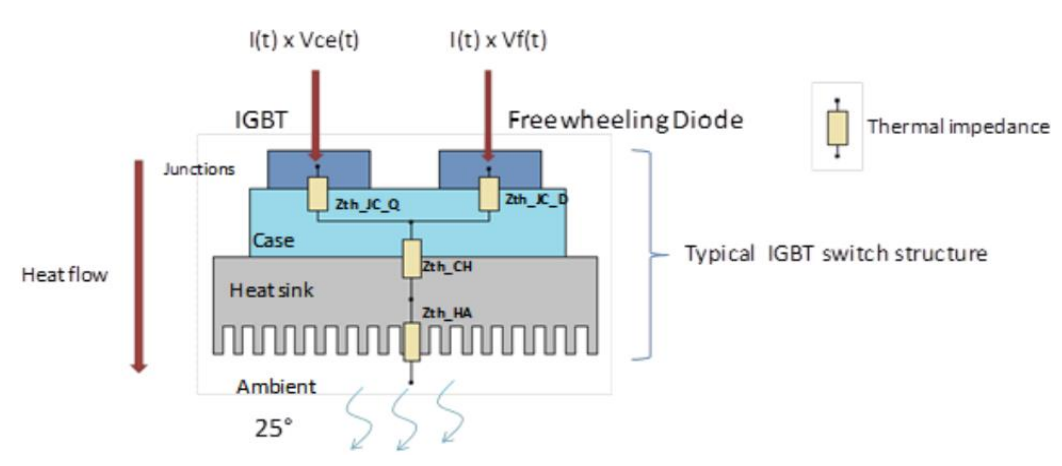

Fig. 6. Estructura simplificada del MOSFET.

El modelo para calcular pérdidas por conmutación y conducción; así, como las temperaturas de unión en el MOSFET y Diodo son a partir de las redes electro-térmica, figura 7. 


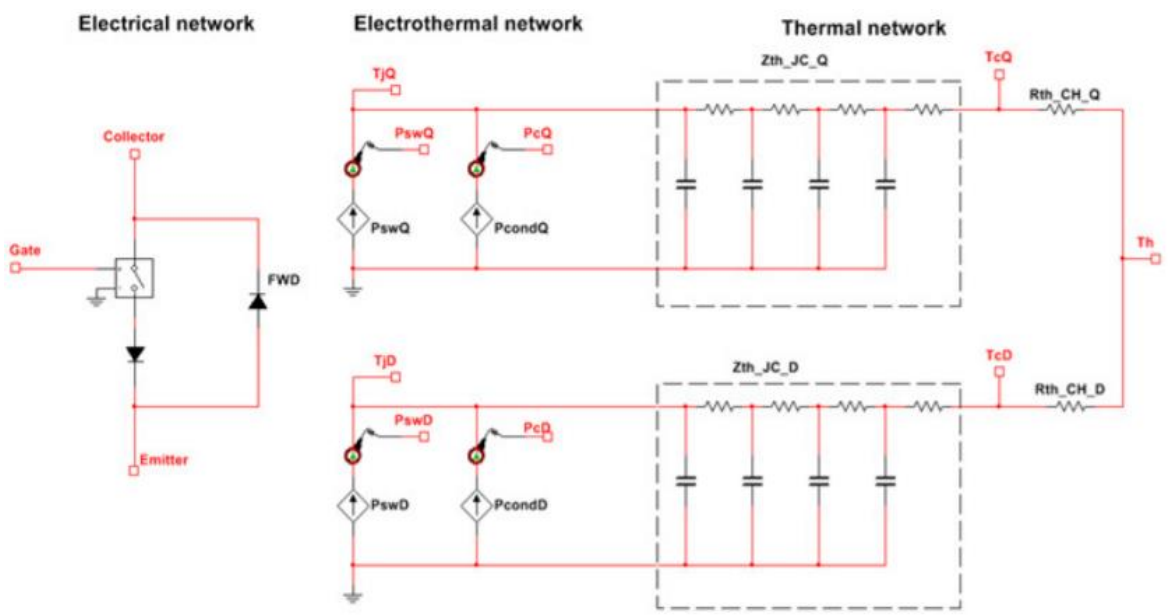

Fig. 7. Modelo térmico del MOSFET-Diodo basado en una red electro-térmica.

\section{Resultados}

La figura 8, muestra el comportamiento de la velocidad del MCDSE utilizando los controladores PID y Difuso, en el arranque, de 0.0 a 0.0013 segundos, los dos controladores presentan el mismo desempeño al llegar a la velocidad de referencia en el mismo tiempo; después del tiempo 0.0013 el controlador difuso presenta major desempeño al mejorar la tolerancia en estado permanente, figura 5 .

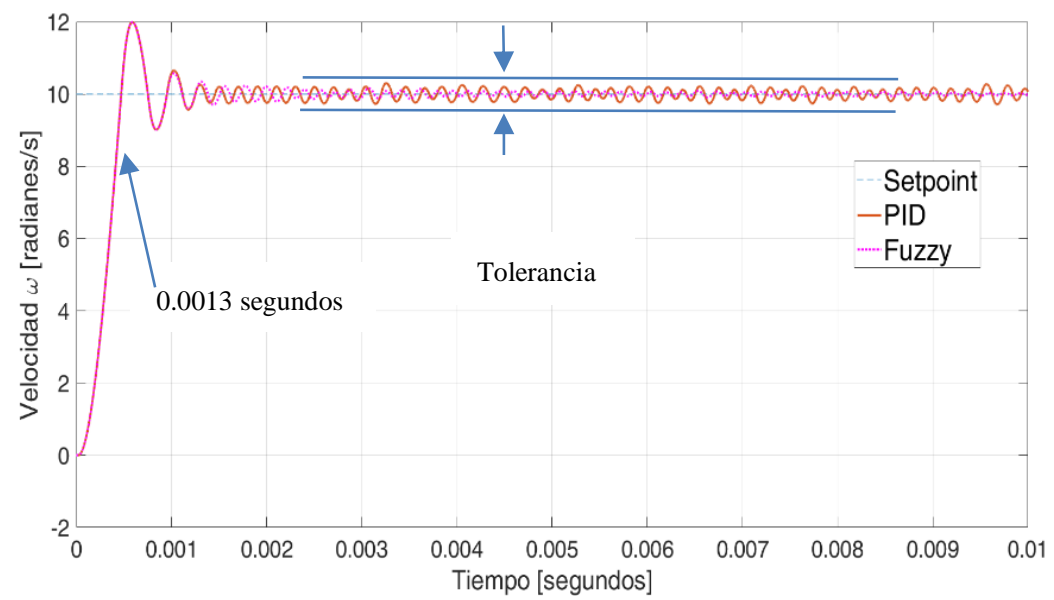

Fig. 8. Respuesta de seguimiento de velocidad. 
La figura 9, muestra las pérdidas por conmutación del MOSFET usando los dos controles y son iguales hasta que el MCDSE llega a la referencia. En estado permanente, las pérdidas son mayores cuando MCDSE en estado permanente con el control PID; mientras que con el control difuso son menores.

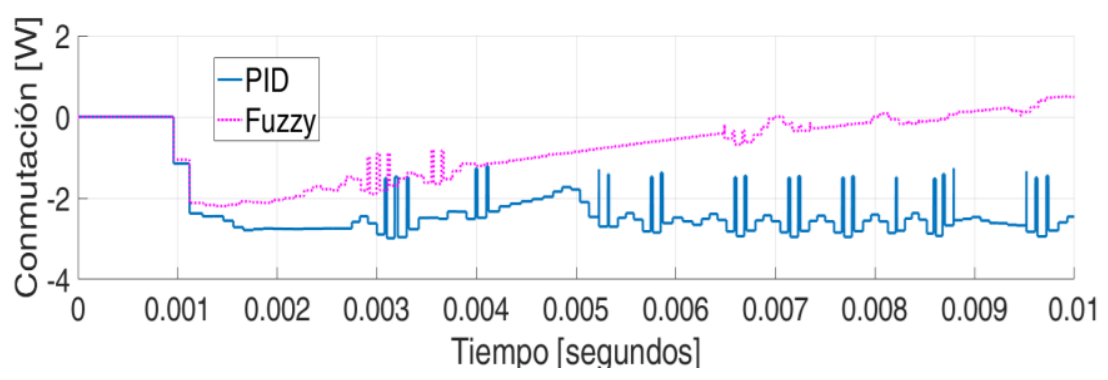

Fig. 9. Perdidas por conmutación del IGBT.

La figura 10, muestra las perdidas por conducción del MOSFET usando los dos controles y son iguale hasta que el MCDSE llega a la referencia. Las pérdidas son mayores cuando MCDSE en estado permanente con el control PID; mientras que con el control difuso son menores.

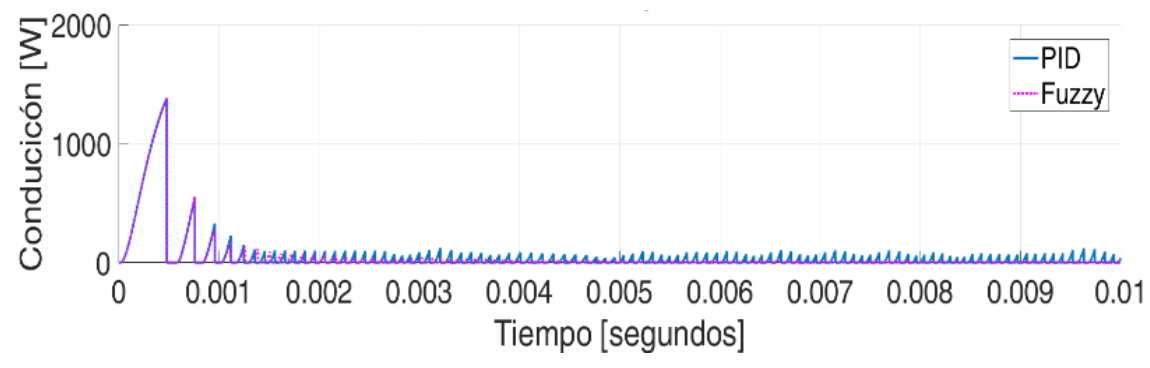

Fig. 10. Perdidas por conducción del IGBT.

La grafica de la figura 11, demuestra el desempeño de los dos controladores basándose en el índice cuadrático del error (ISE); por lo que se infiere que el controlador difuso está más cercano a la referencia ya que tiene un ISE menor en estado permanente que el controlador PID, este hecho en conjunto con los resultados de las pedidas por conducción y conmutación permite deducir que el controlador difuso no solo abate las perdidas sino que también mejora el seguimiento de velocidad, por lo tanto puede atacar dos problemas a la vez. 


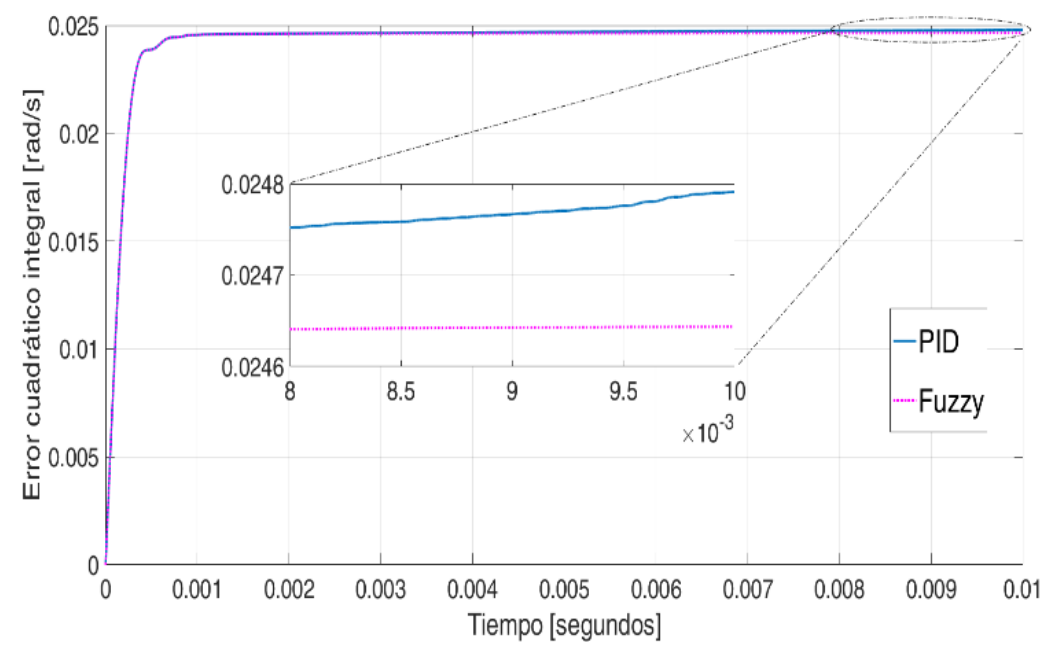

Fig. 11. Índice cuadrático del error (ISE).

\section{Conclusiones}

Este trabajo demuestra la influencia de los esquemas de control PID (controlador lineal) e Inteligente (controlador no-lineal difuso) en las perdidas de conmutación y de conducción en los semiconductores (MOSFET's) de un puente inversor trifásico fuente de voltaje que alimenta a un motor de corriente directa sin escobillas. Otro hecho que se observa que el controlador difuso puede a tacar dos problemas como es de abatir las pérdida y el de estar más cercano a la referencia (reducir el error) en estado permanente. Los resultados obtenidos se validan vía co-simulación Labview-Multsim. Las pérdidas por conmutación y conducción son un factor importante que determinan el estrés por temperatura en los semiconductores de potencia y por consiguiente su vida útil por lo que esta investigación permitirá estudiar la longevidad considerando las estrategias de control.

\section{Referencias}

1. Xia, C. L.: Permanent magnet brushless dc motor drives and controls. John Wiley \& Sons, Singapore (2012)

2. Premkumar, K., Manikandan, B. V.: Speed control of Brushless DC motor using bat algorithm optimized Adaptive Neuro-Fuzzy Inference System. Applied Soft Computing, Vol. 32, pp. 403-419 (2015) 
3. Aström, K. J., Hägglund, T.: Advanced PID control. ISA: The Instrumentation, Systems, and Automation Society (2006)

4. Precup, R. E., Hellendoorn, H.: A survey on industrial applications of fuzzy control. Computers in Industry, Vol. 62, pp. 213-226 (2011)

5. Piris-Botalla, L., Oggier, G. G., Airabella, A. M., García, G. O.: Power losses evaluation of a bidirectional three-port DC-DC converter for hybrid electric system. Electrical Power and Energy Systems, Vol. 58, pp. 1-8 (2014)

6. Hegasy, O., Van-Mierlo, J., Lataire, P.: Analysis, Modeling, and Implementation of a Multidevice Interleaved DC/DC Converter for Fuel Cell Hybrid Electric Vehicles. IEEE Transactions on power electronics, Vol. 27, No. 11, pp. 445-4458 (2012)

7. Bhor, D., Angappan, K., Sivalingam, K. M.: Network and power-grid co-simulation framework for Smart Grid wide-area monitoring networks. Journal of Network and Computer Applications, Vol. 59, pp. 274-284 (2016)

8. Krishnan, R.: Electric Motor Drives: Modeling, analysis and control. Prentice Hall (2001)

9. Andrzej, P.: Fuzzy Modeling and control. Studies in fuzziness and soft computing, Springer-Verlag Berlin Heidelberg, Vol. 69 (2001)

10. Introducción a la Co-Simulación Digital y Analógica entre NI LabVIEW Y NI. Disponible en: Multisim: http://www.ni.com/white-paper/13663/es

11. Power Electronics Development Center. Disponible en: http://forums.ni.com/t5/ Power-Electronics-Development/How-to-simulate-the-temperature-energy efficiency -and-lifetime/gpm-p/3450019

12. Johnson, M. A., Moradi, M. H.: PID Cntrol: New Identification and Design Methods. Springer-Verlag London Limited (2005)

13. Brushless DC machine hall. Disponible en https://beta.multisim.com/help/ components/brushless-dc-machine-hall

14. EPC2015C-Enhancement Mode Power Transistor. Disponible en: www.epc-co.com/ Products/eGaNFETs/EPC2015C.aspx 